\title{
Overview of enabling technologies for 3GPP LTE-advanced
}

\author{
Thien-Toan Tran, Yoan Shin and Oh-Soon Shin
}

\begin{abstract}
As the long term evolution (LTE) standard comes to an end, 3rd Generation Partnership Project is discussing further evolution of the LTE to meet the international mobile telecommunications advanced requirements, which is referred to as LTE-Advanced (LTE release 10 and beyond). This article first presents the network infrastructure of the LTE-Advanced, and then provides an in-depth overview of enabling technologies from the physical layer aspects, including carrier aggregation, advanced multiple-input multiple-output (MIMO) techniques, wireless relays, enhanced inter-cell interference coordination (elCIC), and coordinated multipoint (CoMP) transmission/reception. In particular, we describe concept and principle of each technology and elaborate important technical details. Moreover, we discuss promising study items of the LTE-Advanced for further enhancement.
\end{abstract}

\section{Introduction}

In the last few years, telecommunication industry has been growing explosively. The beginning of modern telecommunication was marked by the invention of telegraph in the eighteenth century. At that time, the message transmission was performed in a rudimentary way, in that there was no mechanism for automatic error correction and interference management. However, people had used it for about 100 years, until Thomas Edison invented telephone in 1876. The telephone network, known as public switched telephone network (PSTN), was rapidly established in Western counties. From that moment, a lot of novel concept and techniques, such as modulation, demodulation, and cellular concept, had been developed to improve the quality of telecommunication services. These inventions constitute the cornerstone of the first mobile telephone service which was introduced by America in 1946 [1,2].

In 1979, the first (1G) commercial cellular network was launched in Japan based on the Nordisk MobilTelefoni (NMT) standard which had been developed in the north European countries. After then, several countries followed, including US, UK, and Canada. In particular, the US deployed an analog cellular standard, called advanced mobile phone system (AMPS), in 1983 and it was widespread over North America [1,2]. A digital cellular standard known as global system for mobile communications

\footnotetext{
* Correspondence: osshin@ssu.ac.kr

School of Electronic Engineering, Soongsil University, Seoul 156-743, Korea
}

(GSM, originally groupe special mobile) was founded in 1987 and became the standard for the 2nd generation (2G) cellular system, improving modulation, voice codecs and security service as compared to $1 \mathrm{G}$ analog systems [3]. In addition to the transition from analog to digital, the GSM system adopted time-division multiple access (TDMA) to serve multiple subscribers, while the AMPS used frequency division multiple access (FDMA). In another side, Qualcomm developed Interim Standard 95 (IS-95) system based on code division multiple access (CDMA) technology [4], which was considered as a part of $2 \mathrm{G}$ systems and was deployed in North America and Korea.

The 3rd Generation Partnership Project (3GPP) and 3GPP2 were founded in 1998 from groups of telecommunication associations to develop the 3rd generation (3G) cellular standard based on CDMA technology. The 3GPP has worked standardization based on the GSM network, whereas the 3GPP2 has continued to evolve the IS-95 network. The standardization had produced two different radio access technologies (RAT's): Wideband CDMA (WCDMA) for 3GPP and cdma2000 for 3GPP2 [5]. The biggest difference is that the WCDMA supports inter-cell asynchronous operation, while the cdma2000 requires inter-cell synchronism relying on the global positioning system (GPS). Since the 3GPP released the first version of the standard, referred to as release 99 or Universal Mobile Telecommunication System (UMTS), in 2000, the standardization have been continuing evolution up to 
now. Accordingly, the peak data rate has increased steeply, from 384/128 kbps for downlink (DL) and uplink (UL) in UMTS, 14/5.7 Mbps in high speed packet access (HSPA), to 28/11 Mbps in HSPA+ [6].

As the CDMA based network reached its limit in accommodating rapidly increasing demand for wireless data traffic, 3GPP decided to develop a standard based on a new access technology, which is called long term evolution (LTE). Especially, the LTE adopted orthogonal frequency division multiplexing (OFDM) instead of CDMA as multiple access technology, in order to efficiently support wideband transmission. In addition, the use of multiple-input multiple-output (MIMO) techniques plays an important role in improving the spectral efficiency. The LTE standard has been finalized, yielding release 9 as its final version. The peak data rate of LTE is as much as 100/50 Mbps for the DL and UL. Since March 2008, 3GPP has been working on further enhancement of the LTE to meet the International Mobile Telecommunications (IMT)-Advanced requirements, which was defined by International Telecommunications Union (ITU) for the fourth generation (4G) evolution [7]. The evolved versions (LTE release 10 and beyond) are called LTE-Advanced [8].

The LTE-Advanced established its own requirements [9], which are tabulated in Table 1 along with International Mobile Telecommunications-Advanced requirements [10]. Most of the LTE-Advanced specifications are set to meet or exceed the IMT-Advanced requirements, which makes the LTE-Advanced a strong candidate for the IMT-Advanced system. In 2009, the 3GPP partners made a formal submission to the ITU proposing that LTE-Advanced be evaluated as a candidate for IMTAdvanced [11]. Important technologies of the LTE-
Advanced for meeting the IMT-Advanced requirements include carrier aggregation, advanced MIMO techniques, wireless relays, enhanced inter-cell interference coordination (eICIC), and coordinated multipoint (CoMP) transmission/reception.

The rest of this article is organized as follows. In Section 2, the network architecture and protocol stack of the LTE-Advanced are described with special focus on the physical layer (PHY). Section 3 presents an overview of several enabling technologies that make the LTEAdvanced meet the IMT-Advanced requirements and be differentiated from the legacy LTE. Finally, in Section 4, concluding remarks are given by identifying promising future work items of the LTE-Advanced.

\section{Network infrastructure}

\subsection{Network architecture}

The core network of the LTE-Advanced system is separated into many parts. Figure 1 shows how each component in the LTE-Advanced network is connected to one another [12-14]. NodeB in $3 G$ system was replaced by evolved NodeB (eNB), which is a combination of NodeB and radio network controller (RNC). The eNB communicates with User Equipments (UE's) and can serve one or several cells at one time. Home eNB (HeNB) is also considered to serve a femtocell that covers a small indoor area. The evolved packet core (EPC) comprises of the following four components. The serving gateway (S-GW) is responsible for routing and forwarding packets between UE's and packet data network (PDN) and charging. In addition, it serves as a mobility anchor point for handover. The mobility management entity (MME) manages UE access and mobility, and establishes the bearer path for

Table 1 Requirements of the IMT-Advanced and LTE-Advanced

\begin{tabular}{|c|c|c|}
\hline Performance metrics & IMT-Advanced requirements & LTE-Advanced requirements \\
\hline Peak data rate & DL 1 Gbps, UL 1 Gbps & DL 1 Gbps, UL 0.5 Gbps \\
\hline Peak spectral efficiency & $\mathrm{DL} 15 \mathrm{bps} / \mathrm{Hz}, \mathrm{UL} 6.75 \mathrm{bps} / \mathrm{Hz}$ & $\mathrm{DL} 30 \mathrm{bps} / \mathrm{Hz}, \mathrm{UL} 15 \mathrm{bps} / \mathrm{Hz}$ \\
\hline Bandwidth & $\begin{array}{l}\text { Scalable bandwidth, minimum } \\
40 \mathrm{MHz}\end{array}$ & Scalable bandwidth, 1.4/3/5/10/15/20 MHz per band, up to total $100 \mathrm{MHz}$ \\
\hline \multicolumn{3}{|l|}{ Latency } \\
\hline User plane & Maximum $10 \mathrm{~ms}$ & Maximum $10 \mathrm{~ms}$ \\
\hline Control plane & Maximum 100 ms & Maximum 50 ms \\
\hline \multicolumn{3}{|l|}{ Handover interrupt time } \\
\hline Intra-frequency & $27.5 \mathrm{~ms}$ & Better than LTE release 8 \\
\hline \multirow[t]{2}{*}{ Inter-frequency } & 40 ms (within a band) & \\
\hline & 60 ms (between bands) & \\
\hline \multicolumn{3}{|l|}{ VolP capacity } \\
\hline Indoor & 50 users/sector/MHz & Better than LTE release 8 \\
\hline Microcell & 40 users/sector/MHz & \\
\hline Base coverage urban & 40 users/sector/MHz & \\
\hline High speed & 30 users/sector/MHz & \\
\hline
\end{tabular}




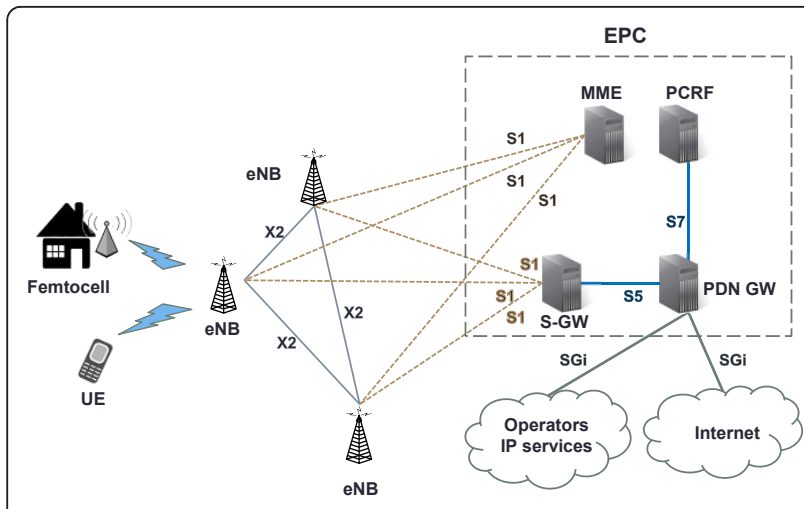

Figure 1 LTE-Advanced network architecture.

UE's. packet data network gateway (PDN GW) is a gateway to the PDN, and policy and charging rules function (PCRF) manages policy and charging rules.

\subsection{Protocol stack}

Protocol stacks for the control plane and user plane are illustrated in Figure 2 [12]. The protocol for user plane includes package data convergence protocol (PDCP), radio link control (RLC), medium access control (MAC), and PHY protocol. The control plane stack additionally includes the radio resource control (RRC) and nonaccess stratum (NAS). The functions of each protocol are summarized in Table 2.

\subsection{Physical layer}

The LTE-Advanced inherits most of specifications from the LTE, but improves them to satisfy the IMTAdvanced requirements. DL multiple access scheme is orthogonal OFDMA, which allows assigning s subset of subcarriers to each individual user. The use of OFDMA provides high flexibility in the number of simultaneously served users and heterogeneous data rates for different users. On the other hand, single carrier-FDMA (SCFDMA) is adopted for the UL. The reason for this is that the SC-FDMA provides much better peak-to-average power ratio (PAPR) property than the OFDMA $[15,16]$, and that the UL transmission usually does not require such high data rate as the DL transmission. Actually, the original SC-FDMA was slightly modified in the LTE-Advanced to support carrier aggregation in the UL, which will be discussed in Section 3.1.

As depicted in Figure 3, the frame length of the LTEAdvanced is $10 \mathrm{~ms}$, which is divided into ten subframes [17]. Each subframe consists of two slots of length $0.5 \mathrm{~ms}$, and each slot contains $N_{\text {symb }}(=7)$ OFDM symbols for the case of normal cyclic prefix. A group of $N_{\mathrm{sc}}^{\mathrm{RB}}(=12)$ adjacent subcarriers constitute a resource block (RB). The larger the transmission bandwidth is, the larger number of RB's ( $=N_{\mathrm{RB}}$ ) will be available. For a bandwidth of $20 \mathrm{MHz}$, for instance, there are 100 RB's available. Differently from the LTE that uses only cell-specific reference signal (CRS), the LTE-Advanced employs UE-specific demodulation reference signal (DM-RS), channel state information reference signal (CSI-RS), multimedia broadcasting single frequency network (MBSFN) reference signal, and positioning reference signal in addition to the CRS [17]. Using these reference signals, LTE-Advanced could support $2 \times 2,4 \times 2,4 \times 4$, and $8 \times 8$ antenna configurations. Control channels for the DL subframe are physical control format indicator channel (PCFICH), physical hybrid automatic repeat request indicator channel $(\mathrm{PHICH})$ and physical DL control channel (PDCCH) [17]. The structure of

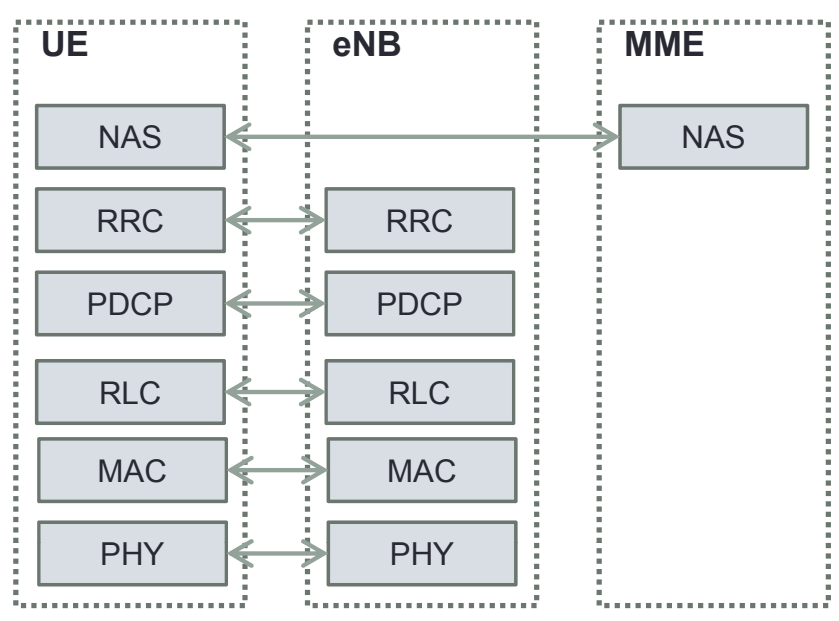

(a)

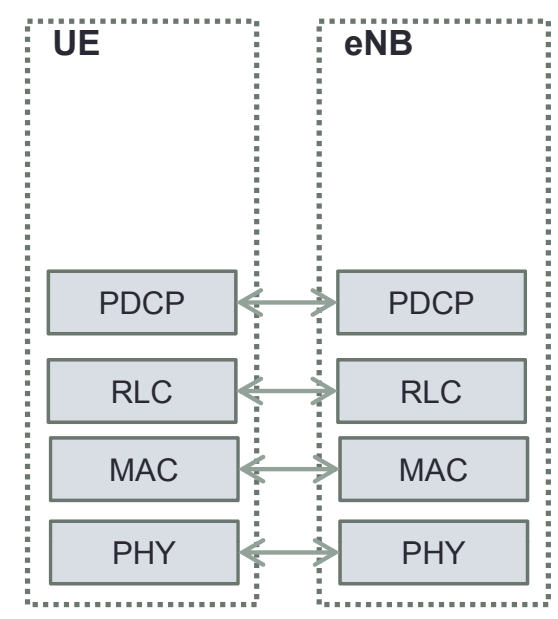

(b)

Figure 2 Protocol stack [12,13]. (a) Control plane. (b) User plane. 
Table 2 Functions of each protocol

\begin{tabular}{|c|c|}
\hline Protocol & Functions \\
\hline$\overline{N A S}$ & $\begin{array}{l}\text { - Connection and session management between UE and the core network } \\
\text { - Authentication } \\
\text { - Registration } \\
\text { - Bearer context activation/deactivation } \\
\text { - Location registration management }\end{array}$ \\
\hline RRC & $\begin{array}{l}\text { - Broadcast system information related to Non-Access Stratum (NAS) and Access Stratum (AS) } \\
\text { - Establishment, maintenance, and release of RRC connection } \\
\text { - Security functions including key management } \\
\text { - Mobility functions } \\
\text { - QoS management functions } \\
\text { - UE measurement reporting and control of the reporting } \\
\text { - NAS direct message transfer between UE and NAS }\end{array}$ \\
\hline PDCP & $\begin{array}{l}\text { - Header compression } \\
\text { - In-sequence delivery and retransmission of PDCP Session Data Units (SDU's) for acknowledge mode radio bearer at handover } \\
\text { - Duplicate detection }\end{array}$ \\
\hline RLC & $\begin{array}{l}\text { - Error correction through Automatic Repeat Request (ARQ) } \\
\text { Segmentation according to the size of transport block and re-segmen in case a retransmission is needed } \\
\text { Concatenation of SDU's for the same radio bearer } \\
\text { - Protocol error detection and recovery } \\
\text { - In-sequence delivery }\end{array}$ \\
\hline MAC & $\begin{array}{l}\text { - Multiplexing/demultiplexing of RLC PDU's } \\
\text { Scheduling information reporting } \\
\text { - Error correction through HARQ } \\
\text { - Local channel prioritization } \\
\text { - Padding }\end{array}$ \\
\hline PHY & $\begin{array}{l}\text { Transmission of electric signals } \\
\cdot \text { Modulation } \\
\cdot \text { Line coding } \\
\cdot \text { Synchronization }\end{array}$ \\
\hline
\end{tabular}

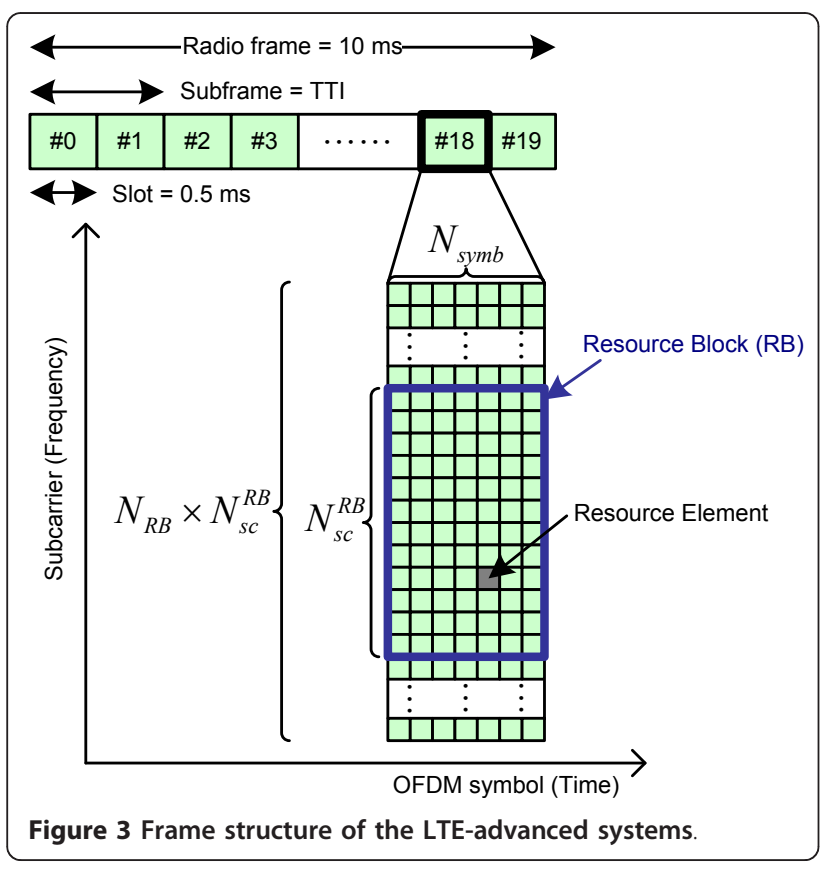

the UL subframe is simpler than that of the DL subframe. Reference signals called demodulation RS's are located within each slot at the fourth OFDM symbol. Only the first and the last subcarriers are dedicated to carry the control signals, such as channel quality indicator (CQI), acknowledgment/negative acknowledgment (ACK/ NACK), and scheduling request indicator (SRI).

\section{Enabling technologies}

\subsection{Carrier aggregation}

According to the ITU-R World Radio communication Conference 2007 (WRC '07), the initial identified spectrum bands for the IMT-Advanced in addition to the already allocated bands are as follows: $450-470 \mathrm{MHz}$, 698-862 MHz, 790-862 MHz, 2.3-2.4 GHz, 3.4-4.2 GHz, and 4.4-4.99 GHz [9]. As a candidate of the IMTAdvanced, the LTE-Advanced should also support those frequency bands. Although the LTE-Advanced can support bandwidth of up to $100 \mathrm{MHz}$, the use of wide bandwidth will be highly limited within available spectrum of operators. In addition, the LTE-Advanced must 
be backward compatible with the legacy LTE, which implies that legacy UE should be able to communicate by using a bandwidth not greater than $20 \mathrm{MHz}$. Carrier aggregation enables both bandwidth extension and backward compatibility by combining several $20 \mathrm{MHz}$ or narrower component carriers (CC's).

As illustrated in Figure 4, there are three types of carrier aggregation: intra-band contiguous carrier aggregation, intra-band non-contiguous carrier aggregation, and inter-band non-contiguous carrier aggregation [18]. The first scenario applies when an operator owns a wide available frequency band. The second scenario will be useful when some unavailable range exists within the LTE-Advanced working bandwidth. The third scenario is the most attractive to operators, since it allows combining CC's distributed across different bands. Possible aggregation scenarios defined in release 10 are summarized in [19].

The original SC-FDMA was designed to work in a contiguous band. In order to support carrier aggregation in the UL as well as the DL, the LTE-Advanced adopts a modified version of SC-FDMA, which is referred to as clustered SC-FDMA. The use of clustered SC-FDMA allows non-contiguous bands for UL transmission, and thus enables frequency selective scheduling within a CC. However, the number of clusters is limited to two in release 10 , since the clustering usually degrades the PAPR performance due to destruction of single carrier characteristic in the time domain [20]. Another aspect of the carrier aggregation is that different sets of CC's can be assigned to the DL and UL. Moreover, even within the same cell, different UE's will work with different numbers of CC's, depending on their capabilities, channel condition, and so on, as illustrated in Figure 5 [11].

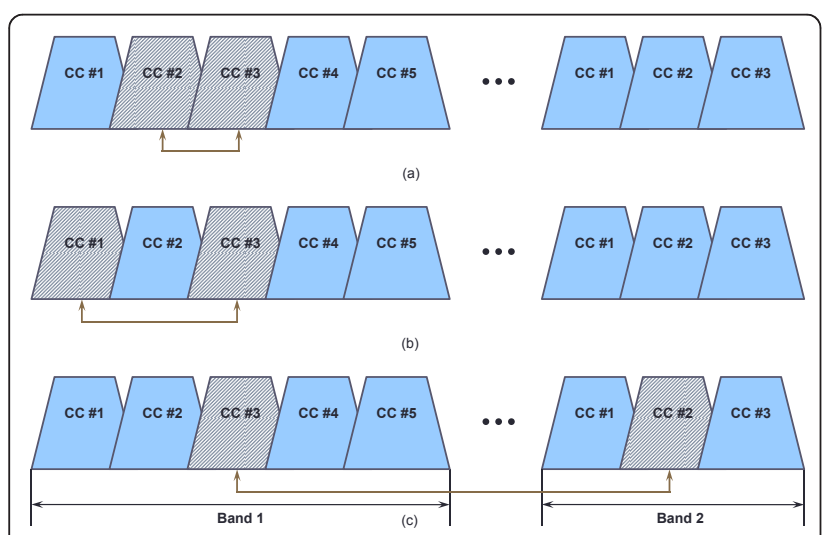

Figure 4 Types of carrier aggregation. (a) Intra-band contiguous carrier aggregation. (b) Intra-band non-contiguous carrier aggregation. (c) Inter-band non-contiguous carrier aggregation.
From the perspective of each UE, CC's are associated to a primary cells (PCell) and possibly plural Secondary Cell (SCell's) [21,22]. The PCell is the only serving cell from which the UE can receive the security input and NAS mobility information for the RRC connection establishment and handover. All the other serving cells, which are allowed at maximum four per UE in release 10, are called SCell's. The PCell assignment is UE-specific, and the change of PCell at each UE can be accomplished only through a handover procedure. The PCell cannot be deactivated, whereas each SCell can be switched on and off dynamically using MAC control element. The PCell is responsible for the monitoring of broadcast signaling of system information and transmission of random access channel (RACH) and PUCCH. The SCell's convey only the dedicated signaling information, $\mathrm{PDSCH}, \mathrm{PUSCH}$, and PDCCH. Meanwhile, the LTE-Advanced is designed to allow cross-carrier scheduling, which means that the PDCCH transmitted from a particular CC may contain the scheduling information on other CC's as well as its own CC. This enables to disperse heavy load of PDCCH transmission in particular CC's.

The idea of carrier aggregation can be generalized such that some LTE-Advanced spectrum can be combined with other frequency bands that are already assigned to other radio RAT's [23]. It is based on the reality that service providers have to pay for their frequency licenses, so they have full authority to use those resources in the most efficient way. The service provider could flexibly select a frequency band among those of several RAT's that are the most appropriate to a specific UE in order to maximize spectrum utilization. Spectrum sharing between different network operators will also be supported [23]. In this scenario, multiple providers will share the common spectrum as well as the network components. Recognizing which operators are available in the cell is one of the additional requirements for UE's. Since each UE selects a specific operator, eNB will forward all data to this operator's network core. This is also called network sharing, and allows operators to reduce the initial investigation cost.

\subsection{Advanced MIMO}

Multiple-input multiple-output refers to a communication system that is equipped with multiple antennas at both transmit and receive sides. The use of MIMO was a key that led to success of IEEE 802.11n, HSPA, and LTE, and now MIMO continues its journey with the LTEAdvanced. According to the LTE-Advanced requirements, the maximum spectral efficiency must be as high as $30 \mathrm{bps} / \mathrm{Hz}$ in the DL, which requires the use of $8 \times 8$ MIMO spatial multiplexing. The DL MIMO was already supported in the LTE in the form of transmit diversity 


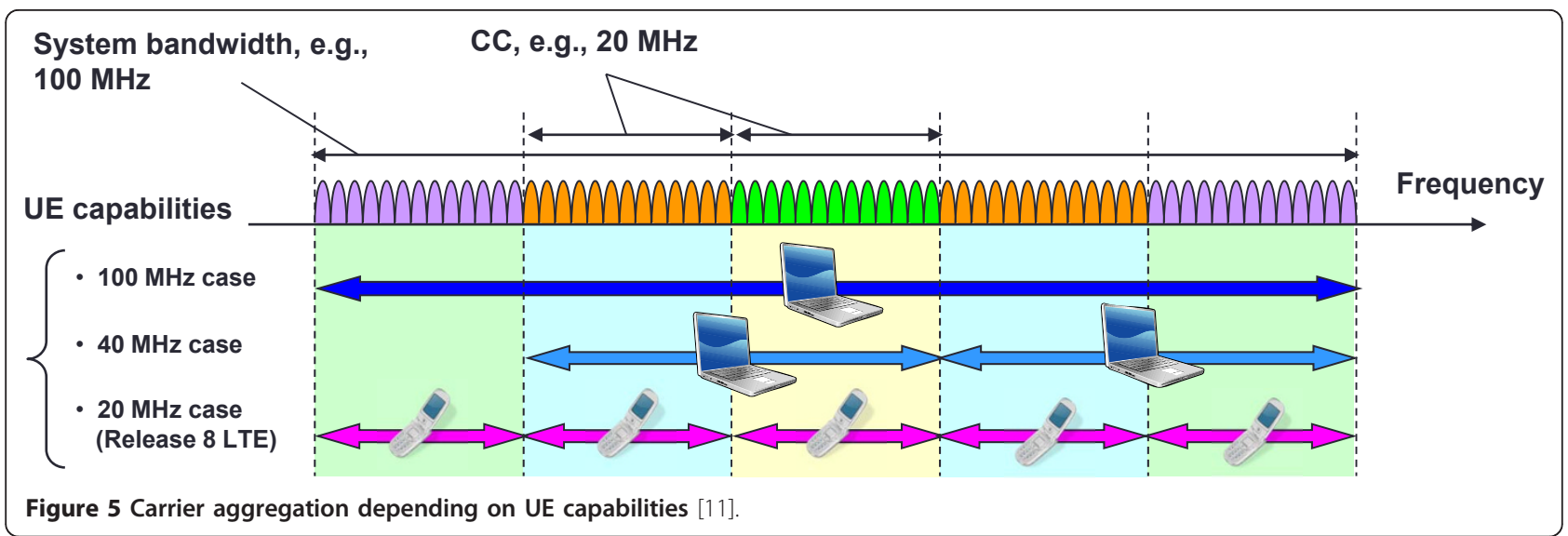

and closed-loop spatial multiplexing up to four layers. One important issue for supporting the $8 \times 8$ MIMO is to design efficient reference signals. The reference signals must be designed in such a way that minimizes the overhead and minimizes the performance degradation due to channel estimation errors at the UE. The LTE employs the CRS for each antenna port, which can be commonly used at every UE. However, the use of CRS for supporting $8 \times 8$ in the LTE-Advanced causes too much overhead. For this reason, the LTE-Advanced employs UEspecific reference signal, called DM-RS, which is used for demodulating data at the corresponding UE. The DM-RS is precoded with the same precoding matrix as that used for the PDSCH transmission, so that the DM-RS cannot be used for generating CQI. Another reference signal, referred to as CSI-RS, is cell-specific and each UE uses it for generating CQI, Precoding Matrix Index (PMI), and rank indicator (RI). Although the CSI-RS is similar to the CRS, the CSI-RS is transmitted much less frequently than the CRS.

The LTE-Advanced adopts a closed-loop precoding to realize $8 \times 8$ MIMO spatial multiplexing. Codebook design is important for closed-loop operation, in that it determines the feedback accuracy and overhead. In the LTE-Advanced, a codebook has been designed based on the assumption of cross-polarized antenna configuration. The resulting codebook can be represented by a product of two matrices as [17]

$$
\mathbf{W}_{1} \mathbf{W}_{2}=\left[\begin{array}{cccccccc}
\mathbf{b}_{i} & \mathbf{b}_{i+1} & \mathbf{b}_{i+2} & \mathbf{b}_{i+3} & \mathbf{0} & \mathbf{0} & \mathbf{0} & \mathbf{0} \\
\mathbf{0} & \mathbf{0} & \mathbf{0} & \mathbf{0} & \mathbf{b}_{i} & \mathbf{b}_{i+1} & \mathbf{b}_{i+2} & \mathbf{b}_{i+3}
\end{array}\right]\left[\begin{array}{c}
\mathbf{e}_{j} \\
\alpha^{H} \mathbf{e}_{j}
\end{array}\right]
$$

where $\mathbf{b}_{i}$ denotes the $i$-th column vector of the extended discrete Fourier transform matrix, $\mathbf{e}_{j}$ denotes 4 $\times 1$ selection vector, i.e., only the $j$-th element is equal to one, while the others are zeroes, and $\alpha$ is the phase difference between horizontal antenna group and vertical antenna group of cross-polarized antennas. The matrix $\mathbf{W}_{1}$ represents wideband and long-term channel characteristics, whereas the matrix $\mathbf{W}_{2}$ represents frequency-selective and short-term channel characteristics. Each UE computes the precoding index and feeds it back to the eNB through either PUCCH or PUSCH. The feedback information includes the CQI and RI as well as the $\mathbf{W}_{1}$ and $\mathbf{W}_{2}$ indexes.

The MIMO transmission is supported in the UL as well. Unlike the LTE that does not support single user MIMO in the UL, the LTE-Advanced supports MIMO with two or four layers. Note that the maximum spectral efficiency of $15 \mathrm{bps} / \mathrm{Hz}$ can be attained only using spatial multiplexing with four layers. The transmission method for reference signals is similar to that of the LTE. The DM-RS is transmitted along with physical UL control channel (PUCCH) and sounding reference signal (SRS) is transmitted at the predefined subframe and symbol. In order to support spatial multiplexing, however, the DMRS of each layer is designed to be orthogonal to one another, using cyclic shifts of a CAZAC (constant amplitude zero auto-correlation) sequence [24]. Moreover, an orthogonal cover code (OCC) is employed to provide additional orthogonality between layers. For the case of the SRS, the orthogonality between layers is realized by using cyclic shifts of the SRS sequence, different frequency combs or combination of the two.

The codebook for UL precoding was designed to preserve the single carrier property of the SC-FDMA. Specifically, the use of Cubic Metric Preserving (CMP) matrix makes each antenna carry signals from only one layer, so that the CM or PAPR is preserved after precoding. In addition, antenna selection matrices are also employed as precoding matrices, since they are useful when the antenna gain imbalance is severe. The resulting UL codebooks for 2 and 4 antennas are shown in Figure 6 [17].

\subsection{Wireless relays}

The eNB has been improved with many powerful functions and plays an important role in the LTE-Advanced 


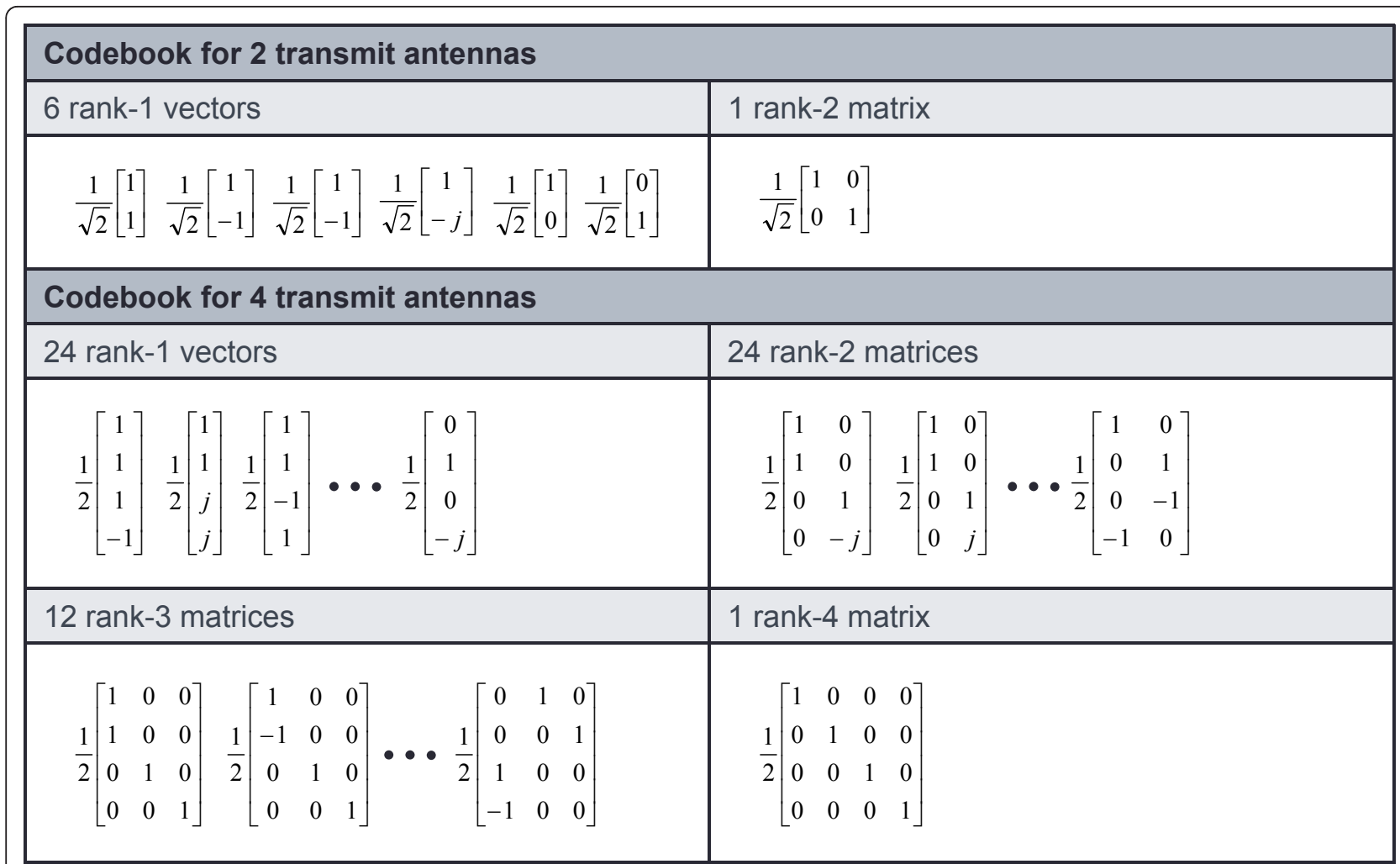

Figure 6 UL codebooks for 2 and 4 transmit antennas.

systems. However, due to that reason, its cost is also getting higher, and could lead to extension trouble. Wireless relaying is another improvement of the LTEAdvanced that can solve such problem. First of all, the use of wireless relays could provide cell coverage extension and cell edge performance improvement. In addition, wireless relays can reduce coverage holes, enhance throughput, and provide group mobility [25].

The relay node $(\mathrm{RN})$ establishes a wireless connection with radio access network via a donor cell. The eNB corresponding to the donor cell is called Donor eNB (DeNB) for the RN. The connection can be either inband or outband. For the case of inband relays, the DeNB-to-RN link share the same band with direct DeNB-to-UE links within the donor cell. This means that the inband relay needs to operate in half-duplex mode to avoid self-interference problem, unless sufficient physical isolation or interference cancellation is secured between transmit antennas and receive antennas at the RN. Moreover, legacy LTE UE's should be able to connect to the donor cell even in this scenario for backward compatibility. In particular, Figure 7 illustrates the DL transmission scenario when an inband RN employs time division for the separation of the DeNB-to-RN and RN-to-UE links, i.e., the two links become active in different subframes. In this case, during the subframe in which the DeNB-to-RN link is active, the RN cannot send any signal, including the CRS and PDCCH. Without the knowledge on the timing of such subframes, however, legacy UE's will not accurately measure the CRS, which will cause significant performance degradation. To solve the problem, the MBSFN signaling defined in LTE release 8 is exploited, as depicted in Figure 7 [26]. In the MBSFN subframe, the CRS is not transmitted except for the first one or two OFDM symbols. Once the RN declares that the a certain subframe is MBSFN subframe, the RN transmits the CRS and PDCCH to UE's during one or two OFDM symbols, and receives from the DeNB in the remaining time of the subframe. Correspondingly, the legacy UE's will attempt to receive the CRS and $\mathrm{PDCCH}$ during only one or two

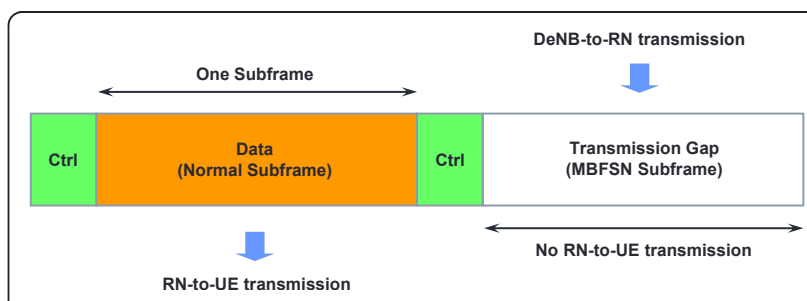

Figure 7 Resource partitioning between the DeNB-to-RN link and RN-to-UE link [26]. 
OFDM symbols in the MBSFN subframe. This method of provisioning backward compatibility is called fake MBSFN, since the RN plays a trick on legacy UE's using the MBSFN signaling and subframe structure. On the other hand, the outband relay is much easier to implement, since the DeNB-to-RN link and RN-to-UE link operate in different bands and thus can easily work in full-duplex mode.

In the LTE-Advanced, two types of inband RN's are defined according to the functions and supportable protocol layers. A Type $1 \mathrm{RN}$ controls its own cell with its own cell identity, including transmission of the CRS. The Type $1 \mathrm{RN}$ appear as if it is an eNB to the legacy UE's, which ensures backward-compatible operation. A Type 2 RN does not have its own cell identity and is part of the donor cell. The Type $2 \mathrm{RN}$ just helps the DeNB and UE's transmit data; control signals are transmitted directly from the eNB to UE's. The functions and characteristics of the Type 1 and Type 2 RN's are summarized in Table 3 [26].

The relaying functionality can be realized in the form of amplify-and-forward or decode-and-forward scheme. In the amplify-forward scheme, relays just transmit an amplified version of the received signal from the source like a traditional repeater. The performance of the scheme is usually limited by noise amplification. In the decode-and-forward scheme, relays decode the received signal and re-encodes it to transmit [27]. The decodeand-forward scheme can be extended to cooperative communication schemes by allowing relays to interwork with the transmitter or receiver [28]. One of the most important challenges in wireless relays is to reduce backhaul overhead caused by the separation of DeNBto-RN and RN-to-UE links, as explained above, which is called half-duplex constraint $[29,30]$. Solutions to this problem include the use of hybrid automatic repeat request (H-ARQ) [31] and partial relaying [32,33], in which only partial information is transmitted through the relay. Other important research issues are MIMO processing at the relay $[34,35]$, and relay selection techniques [36].

\subsection{Enhanced eICIC}

With an explosive growth in wireless traffic, a variety of small-size low-power base stations are being deployed within the usual macro eNB to serve hot zone, office, and home areas. This type of overlay architecture is referred to as heterogeneous network (HetNet) [37-39]. Table 4 shows several types of nodes that may exist in a HetNet [38]. Note that relays discussed in Section 3.3 can also be considered as one part of a HetNet. Different types of nodes are optimized for better coverage and data transmission. For example, a macrocell, equipped with an eNB, covers a large area of a few kilometers and serves thousands of users, while a picocell uses a lowpower type of eNB and serves tens of users in areas where the signal strength from macrocells is weak.

Due to a large number of heterogeneous cells that could exist in a certain area, inter-cell interference becomes a challenging issue in HetNet scenarios. In particular, in certain situations, the signal from the serving cell could be much weaker than that from the interfering cells, which is referred to as dominant interference scenario [38]. Figure 8 illustrates dominant interference scenarios between a macrocell and femtocells. Usually, femtocells adopt closed subscriber group (CSG) policy, which means that only a group of permitted users are allowed to access the femtocell. When a femtocell operates in the CSG mode, some UE's entering a femtocell from macrocell need to keep the connection with a macrocell; they cannot handover to the femtocell even though the signal from the femtocell is much stronger. In this case, the UE's will be affected by strong interference from the femtocell in the DL reception. Also, UE's associated to femtocell will be interfered by macro UE's in the UL transmission.

Table 3 Characteristics of Type 1 and Type 2 RN's

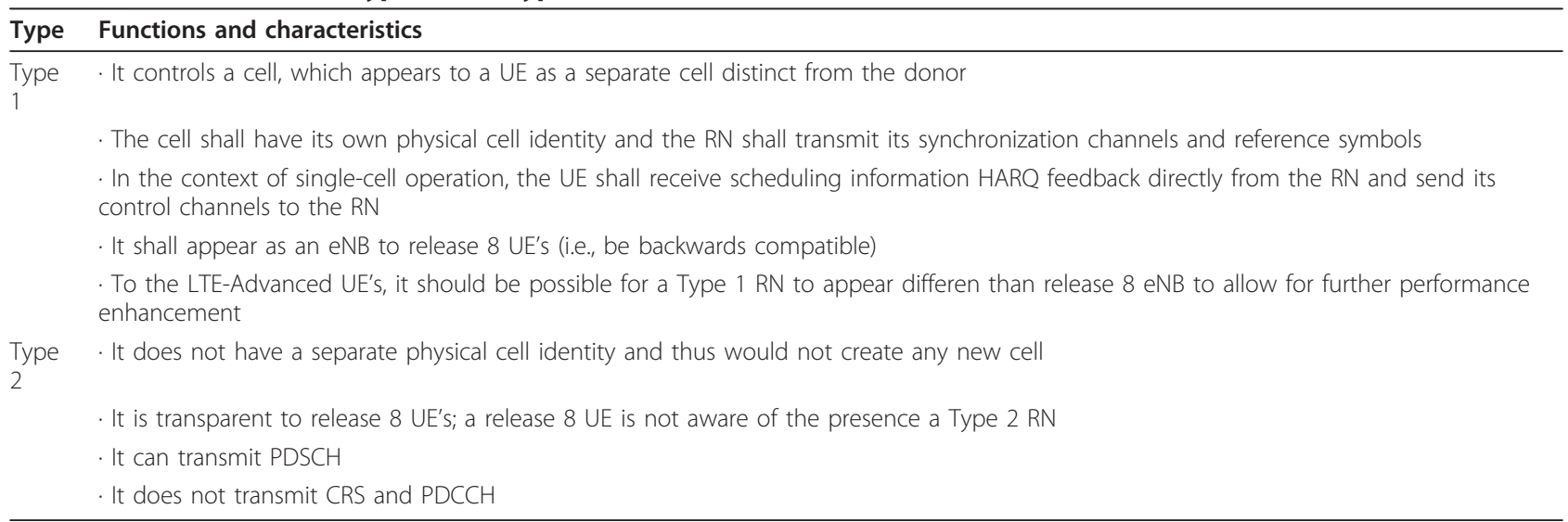


Table 4 Characteristics of several types of nodes in heterogeneous networks

\begin{tabular}{llll}
\hline Type of nodes & Transmit power $(\mathbf{d B m})$ & Coverage & Backhaul \\
\hline Macrocell & 46 & Few $\mathrm{km}$ & S1 interface \\
Picocell & $23-30$ & $<1300 \mathrm{~m}$ & $\times 2$ interface \\
Femtocell & $<23$ & $<50 \mathrm{~m}$ & Internet IP \\
Relay & 30 & $300 \mathrm{~m}$ & Wireless \\
RRH & 46 & Few km & Fiber \\
\hline
\end{tabular}

The LTE release 8 and 9 employ messages for ICIC that can be exchanged between eNB's via the X2 interface, such as the following three indicators [38]: (1) Relative narrowband transmit power (RNTP) indicator is used by a certain cell to inform neighboring cells which DL RB's it is using to serve UE's within and transmit power level for the corresponding RB's. (2) Overload indicator (OI) is used to inform neighboring eNB's on a certain eNB's self-estimated interference level on UL RB's. When other eNB's receive this information, they would attempt to reschedule or reduce activities on those RB's. (3) High interference indicator (HII) allows one eNB to warn neighboring eNB's that certain UL RB's will be heavily loaded in the near future to serve its own celledge UE's. Other eNB's would abstain from using those RB's to avoid mutual interference.

The ICIC methods of the LTE release 8 and 9 do not consider dominant interference scenarios of HetNets. In order to address such scenarios, the LTE-Advanced has been developing enhanced ICIC (eICIC) techniques, which can be classified into three categories [40,41]: time-domain techniques, frequency-domain techniques, and power control techniques. In time-domain techniques, the victim users are scheduled in time-domain resources where interference from other nodes is mitigated. Time-domain techniques employ subframe

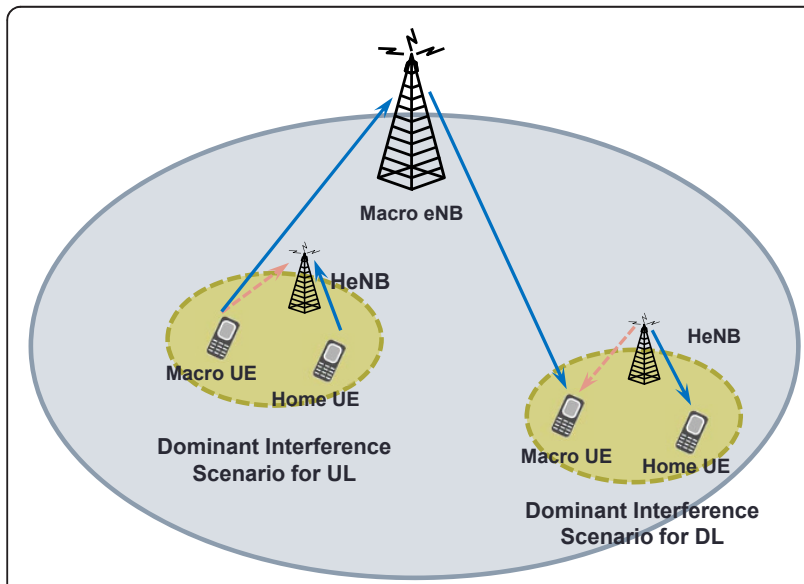

Figure 8 Dominant interference scenarios due to coexistence of macrocells and femtocells. alignment and OFDM symbol shift. In particular, subframe alignment can be realized by cooperative silencing. The aggressor eNB's set almost blank subframe (ABS) where only minimal control signals are transmitted, and inform the eNB with victim UE's of the ABS pattern via the X2 interface or Operation and Management $(\mathrm{O} \& M)$. Then, the eNB can serve the victim UE's during the subframe with reduced interference. In frequency-domain techniques, control and reference signals are scheduled in reduced bandwidth, so that the signals of different cells are ensured to be orthogonal to one another. While frequency-domain orthogonality can be achieved in a static manner, it may also be implemented dynamically through victim UE detection. In power control techniques, femtocells employ power control schemes different from the one used in macrocells. The power control scheme can be designed by accounting for the following factors: the strongest macro eNB received power at a $\mathrm{HeNB}$, path loss between a HeNB and macro UE, target signal-to-interference-plus-noise ratio (SINR) of home UE, and target SINR of macro UE.

\subsection{CoMP transmission/reception}

Carrier aggregation and CoMP are the two most important techniques that boost the data rate of the LTEAdvanced to a new threshold. If we call CA a road of the LTE-Advanced, CoMP surely will be a car which the LTE-Advanced drives. In traditional telecommunication systems, each UE will be basically served by only one base station (BS) at a moment. Signals come from other BS's will become interference to the UE. When the UE moves to the cell edge, it will communicate with more than one BS's to prepare for handover. However, it is still being served by its original BS. This is also the time when the UE receives strong interference, and data rate will be very low. The situation will become worse if the UE is moving with high speed.

Coordinated multipoint can be considered as a distributed MIMO system, in that geographically distributed nodes form multiple antennas and they cooperate to transmit to and/or receive from UE's [42-44]. CoMP has been studied as a solution for increasing the system throughput, especially at cell edge areas where inter-cell interference is severe with traditional approach. Due to the potential advantage, CoMP techniques received a lot of attention at the initiatory stage of the LTE-Advanced standardization. However, in practice, there are critical issues in CoMP, such as excessive feedback overhead [45], backhaul delay and burden [46,47], and interference channel estimation [48]. Accordingly, the discussion on CoMP was suspended in release 10, but it is being discussed again in release 11.

Coordinated multipoint can be applied to both the DL and UL. DL CoMP techniques can be classified 


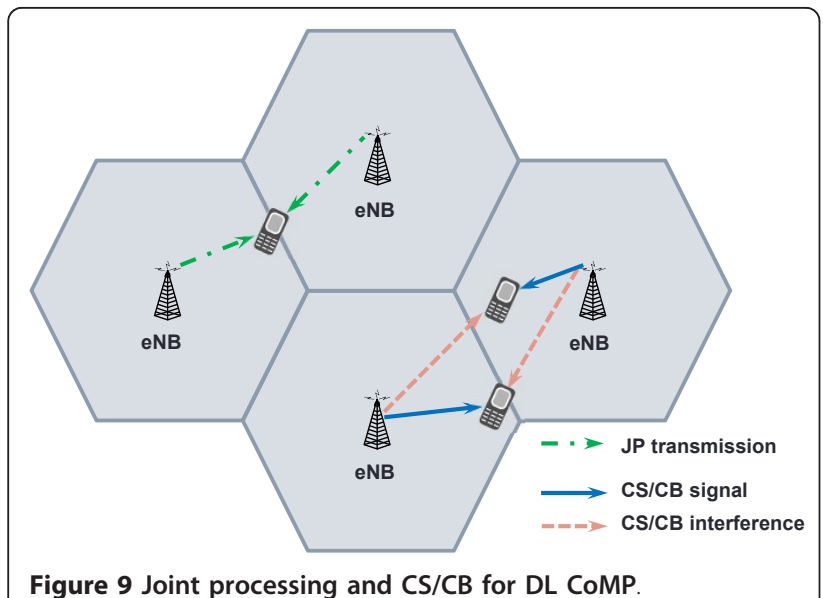

according to the amount of information shared among cells. Joint processing is available when neighboring cells share transmit data as well as the channel state information. The joint processing can be realized in the form of joint transmission or dynamic cell selection. In joint transmission, cooperating eNB's jointly transmit data to one or more corresponding UE's. Dynamic cell selection is a kind of fast cell selection; UE's are handed over to the best cell considering interference situation. However, joint processing generally requires high-capacity $\mathrm{X} 2$ interface between eNB's for sharing transmit data, and thus can cause excessive backhaul overhead and latency. On the other hand, coordinated scheduling/coordinated beamforming $(\mathrm{CS} / \mathrm{CB})$ can be realized only if the channel state information and scheduling information are shared among eNB's [44]; data sharing is not required. In the $\mathrm{CS} / \mathrm{CB}$, a UE receives data from only one eNB, its own serving node, while the precoding and scheduling are coordinated among related eNB's in such a way to reduce interference and improve the throughput. Figure 9 illustrates how the joint processing and $\mathrm{CS} / \mathrm{CB}$ serve UE's at the cell edge.

For the case of UL, joint detection and interference prediction are considered. Joint detection can be considered as a UL counterpart of the DL joint transmission. For joint detection, eNB's need to share received signal samples as well as channel state information and scheduling information. The basic principle of interference prediction is to perform link adaptation based on predicted SINR values [49]. Interference prediction is possible by exchanging resource allocation information among cells. Another emerging UL CoMP scheme of importance is interference-aware distributed precoding $[50,51]$, which can be implemented in fully distributed

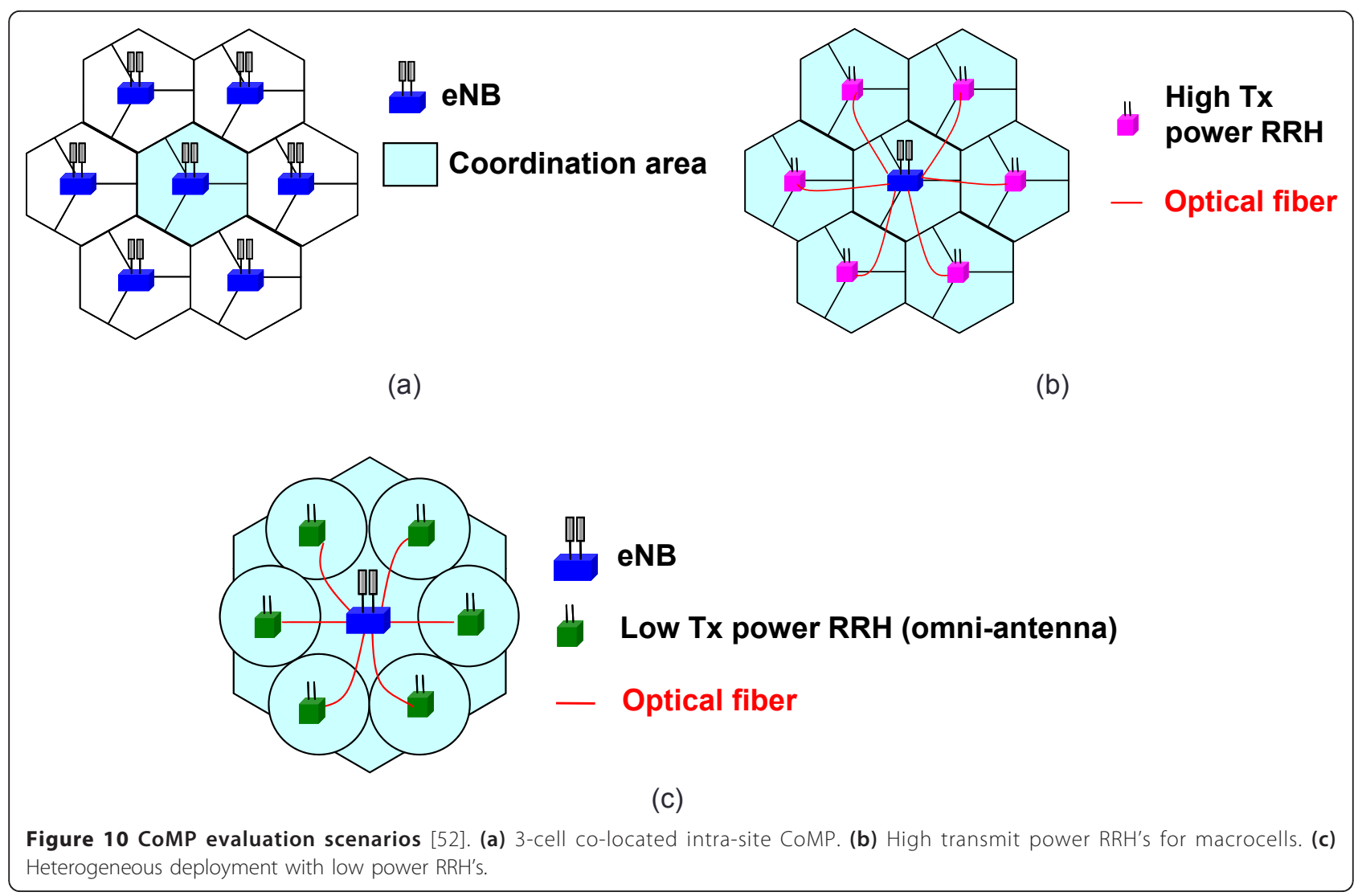


manner without sharing even channel state information among eNB's.

According to R1-110564 in 3GPP, CoMP techniques can be applied in three different scenarios [52], as illustrated in Figure 10. Currently, various CoMP schemes are being evaluated by several institutes under the scenarios. The scenarios of particular interest are the two scenarios with remote radio head (RRH), which ensures high capacity and low latency backhaul.

\section{Conclusion}

This article has provided an overview of current status of advanced technologies that enable the LTE-Advanced to meet its requirements and differentiate it from the legacy LTE. The standardization process on the LTEAdvanced is still ongoing, and more advanced features are expected to be discussed and included in the future. Promising study items include enhanced carrier aggregation without the backward compatibility constraint, efficient MIMO schemes under the scenario that lowpower nodes are nonuniformly distributed, open-loop MIMO scheme with reduced overhead and complexity, mobile relays that can provide group mobility, improved eICIC techniques that can mitigate the residual interference in ABS, and efficient feedback mechanism and codebook design for CoMP operation.

\section{Acknowledgements}

This work was supported in part by the Human Resources Development of the Korea Institute of Energy Technology Evaluation and Planning (KETEP) grant funded by the Korea government Ministry of Knowledge Economy (No. 20114010203110), and in part by the National Research Foundation of Korea (NRF) grant funded by the Korea government (MEST) (No. 20090085604).

\section{Competing interests}

The authors declare that they have no competing interests.

Received: 16 September 2011 Accepted: 20 February 2012 Published: 20 February 2012

\section{References}

1. V Gautam, S Sinha, Understanding Telecom Management, (Concept Publishing Company, New Delhi, 2004), pp. 24-25

2. Wireless Museumhttp://www.wirelessmuseum.org/history/

3. M Mouly, MB Pautet, T Haug, The GSM System for Mobile Communications, (Telecom Publishing, Palaisea, 1992)

4. JS Lee, LE Miller, CDMA Systems Engineering Handbook, (Artech House Publishers, Boston, 1998)

5. T Ojanperä, R Prasad, WCDMA: Towards IP Mobility and Mobile Internet, (Artech House Publishers, Boston, 2001)

6. AM Rao, A Weber, S Gollamudi, R Soni, LTE and HSPA+: revolution-ary and evolutionary solutions for global mobile broadband. Bell Labs Tech J. 13(4), 7-34 (2009). doi:10.1002/bltj.20334

7. Background on IMT-Advanced, ITU-R 5D IMT-ADV/1-E (2008)

8. 3GGP LTE-Advanced official websitehttp://www.3gpp.org/LTE-Advanced/

9. Requirements for further advancements for Evolved Universal Terrestrial Radio Access (E-UTRA) (LTE-Advanced), 3GPP TR 36.913, V10.0.0 (2011)

10. Requirements related to technical performance for IMT-Advanced radio interface(s), Report ITU-R M.2134 (2008)
11. Proposal for candidate radio interface technologies for IMT-Advanced based on LTE release 10 and beyond (LTE-Advanced), 3GPP RP-091005 (2009)

12. Evolved Universal Terrestrial Radio Access (E-UTRA) and Evolved Universal Terrestrial Radio Access Network (E-UTRAN); Overall description, 3GPP TS 36.300, V11.0.0 (2011)

13. Long Term Evolution (LTE), A technical overview, Motorola, Tech. White Paper.

14. IF Akyildiz, DM Gutierrez-Estevez, EC Reyes, The evolution to 4 G cellular systems: LTE-Advanced. Phys Commun. 3, 217-244 (2010). doi:10.1016/j. phycom.2010.08.001

15. HG Myung, J Lim, DJ Goodman, Single carrier FDMA for uplink wireless transmission. IEEE Veh Technol Mag. 1(3), 30-38 (2006)

16. HG Myung, J Lim, DJ Goodman, Peak-to-average power ratio of single carrier FDMA signals with pulse shaping, in Proceeding of the IEEE 17th International Symposium on Personal, Indoor and Mobile Radio Communications, Helsinki, Finland, pp. 1-5. 11-14 Sept (2006)

17. Evolved Universal Terrestrial Radio Access (E-UTRA); Physical channels and modulation, 3GPP TS 36.211, V10.2.0 (2011)

18. M Iwamura, K Etemad, MH Fong, R Nory, R Love, Carrier aggregation framework in 3GPP LTE-advanced. IEEE Commun Mag. 48(8), 60-67 (2010)

19. Evolved Universal Terrestrial Radio Access (E-UTRA); User Equipment (UE) radio transmission and reception, 3GPP TR 36.807, V0.1.0 (2010)

20. M Rumney, 3GPP LTE/LTE-A standardization: status and overview of technologies, in Proceeding of the Future Network and Mobile Summit, Warsaw, Poland, pp. 1-33. (14-17 June 2011)

21. Evolved Universal Terrestrial Radio Access (E-UTRA); Medium access control (MAC) protocol specification, 3GPP TS 36.321, V10.4.0 (2011)

22. KI Pedersen, F Frederiksen, C Rosa, H Nguyen, LGU Garcia, Y Wang, Carrier aggregation for LTE-advanced: functionality and performance aspects. IEEE Commun Mag. 49(6), 89-95 (2011)

23. Network sharing, architecture and functional description, 3GPP TS 23.251, V10.2.0 (2011)

24. BM Popovic, Generalized chirp-like polyphase sequences with optimal correlation properties. IEEE Trans Inf Theory. 38(7), 1406-1409 (1992)

25. Y Yang, $\mathrm{H} \mathrm{Hu}, J \mathrm{Xu}, \mathrm{G}$ Mao, Relay technologies for WiMAX and LTEadvanced mobile systems. IEEE Commun Mag. 47(10), 100-105 (2009)

26. Evolved Universal Terrestrial Radio Access (E-UTRA); Further advance-ments for E-UTRA physical layer aspects, 3GPP TR 36.814, V9.0.0 (2010)

27. RU Nabar, H Bölcskei, FW Kneubühner, Fading relay channels: performance limits and space-time signal design. IEEE J Sel Areas Commun. 22(8), 1099-1109 (2004)

28. A Nosratinia, TE Hunter, A Hedayat, Cooperative communication in wireless networks. IEEE Commun Mag. 42(10), 74-80 (2004). doi:10.1109/ MCOM.2004.1341264

29. JN Laneman, DNC Tse, GW Wornell, Cooperative diversity in wireless networks: efficient protocols and outage behavior. IEEE Trans Inf Theory. 50(12), 3062-3080 (2004). doi:10.1109/TIT.2004.838089

30. D Chen, JN Laneman, Modulation and demodulation for cooperative diversity in wireless systems. IEEE Trans Wirel Commun. 5(7), 1785-1794 (2006)

31. B Zhao, MC Valenti, Practical relay networks: a generalization of hybrid-ARQ. IEEE J Sel Areas Commun. 23(1), 7-18 (2005)

32. OS Shin, JH Lee, Efficient partial relaying protocol for wireless multihop transmission. Wirel Pers Commun (2011). doi:10.1007/s11277-011-0266-y

33. DI Kim, W Choi, H Seo, BH Kim, Partial information relaying with per antenna superposition coding. IEEE Trans Commun. 58(12), 3423-3427 (2010)

34. B Wang, J Zhang, A Høst-Madsen, On the capacity of MIMO relay channels. IEEE Trans Inf Theory. 51(1), 29-43 (2005)

35. AS Behbahani, R Merched, AM Eltawil, Optimizations of a MIMO relay network. IEEE Trans Signal Process. 56(10), 5062-5073 (2008)

36. $Y$ Jing, $H$ Jafarkhani, Single and multiple relay selection schemes and their achievable diversity orders. IEEE Trans Wirel Commun. 8(3), 1414-1423 (2009)

37. A Damnjanovic, J Montojo, Yongbin Wei, Tingfang Ji, Tao Luo, M Vajapeyam, Taesang Yoo, Osok Song, D Malladi, A survey on 3GPP heterogeneous networks. IEEE Wirel Commun. 18(3), 10-21 (2011)

38. D Lopez-Perez, A Valcarce, G De La Roche, J Zhang, Enhanced intercell interference coordination challenges in heterogeneous networks. IEEE Wirel Commun. 18(3), 22-30 (2011) 
39. D Lopez, A Valcarce, GDL Roche, J Zhang, OFDMA femtocells: A roadmap on interference avoidance. IEEE Commun Mag. 47(9), 41-48 (2009)

40. Summary of the description of candidate elCIC solutions, 3GPP R1-104968 (2010)

41. A Valcarce, GDL Roche, A Juttner, D Lopez, J Zhang, Applying FDTD to the coverage prediction of WiMAX femtocells. EURASIP J Wirel Commun Netw (2009). 2009, 13 Article ID 308606

42. M Sawahashi, Y Kishiyama, A Morimoto, D Nishkawa, M Tanno, Coordinated multipoint transmission/reception techniques for LTE-Advanced. IEEE Wirel Commun. 17(3), 26-34 (2010)

43. J Zhang, R Chen, JG Andrews, A Ghosh, RW Heath, Networked MIMO with clustered linear precoding. IEEE Trans Wirel Commun. 8(4), 1910-1921 (2009)

44. H Dahrouj, W Yu, Coordinated beamforming for the multicell multiantenna wireless system. IEEE Trans Wirel Commun. 9(5), 1748-1759 (2010)

45. D Kim, OS Shin, KB Lee, Efficient limited feedback schemes for network MIMO systems, in Proceedings of the IEEE 54th Global Communications Conference, Houston, TX, USA, pp. 502-507. (5-9 Dec 2011)

46. O Simeone, O Somekh, HV Poor, S Shamai, Downlink multicell processing with limited backhaul capacity. EURASIP J Adv Signal Process (2009). (Article ID 840814)

47. R Zakhour, D Gesbert, Optimized data sharing in multicell MIMO with finite backhaul capacity. IEEE Trans Signal Process. 59(12), 6102-6111 (2011)

48. Y Ohwatari, N Miki, T Abe, S Nagata, Y Okumura, Investigation on improvement in channel estimation accuracy using data signal muting in downlink coordinated multiple-point transmission and reception in LTEAdvanced, in Proceedings of the IEEE Wireless Communications and Networking Conference, Quintana-Roo, Mexico, pp. 1288-1293. (28-31 Mar 2011)

49. A Müller, P Frank, Cooperative interference prediction for enhanced link adaptation in the 3GPP LTE uplink. in Proceedings of the IEEE 71st Vehicular Technology Conference-Spring 1-5. Taipei, (16-19 May 2010)

50. D Gesbert, S Hanly, H Huang, S Shamai, O Simeone, W Yu, Multi-cell MIMO cooperative networks: a new look at interference. IEEE J Sel Areas Commun. 28(12), 1-29 (2010)

51. BO Lee, HW Je, OS Shin, KB Lee, A novel uplink MIMO transmission scheme in a multicell environment. IEEE Trans Wirel Commun. 8(10), 4981-4987 (2009)

52. Report from offline discussion on CoMP simulation assumptions. 3GPP R1$110564(2011)$

doi:10.1186/1687-1499-2012-54

Cite this article as: Tran et al:: Overview of enabling technologies for 3GPP LTE-advanced. EURASIP Journal on Wireless Communications and Networking 2012 2012:54.

\section{Submit your manuscript to a SpringerOpen ${ }^{\mathcal{O}}$ journal and benefit from:}

- Convenient online submission

- Rigorous peer review

- Immediate publication on acceptance

- Open access: articles freely available online

- High visibility within the field

- Retaining the copyright to your article

Submit your next manuscript at $\gg$ springeropen.com 